\title{
Des délétions de l'ADN mitochondrial dans le syndrome de Kearns-Sayre et autres myopathies avec ophtalmoplégie externe progressive
}

Le syndrome de Kearns-Sayre associe une myopathie avec ophtalmoplégie externe progressive, une rétinite pigmentaire et des troubles de la conduction myocardique. Des formes plus frustes comportent de façon prédominante l'ophtalmoplégie externe. Histologiquement, la présence de fibres musculaires anormales (en lambeaux) est constante. Ces maladies apparaissent le plus souvent de façon sporadique et sont associées à des délétions d'une partie des molécules de l'ADN mitochondrial, variables d'un malade à l'autre. Ces anomalies du génome mitochondrial sont à l'origine d'anomalies biochimiques reflétant la perturbation des fonctions mitochondriales, c'est-à-dire de la production d'énergie via les réactions de phosphorylation oxydative.

\section{Isabelle Nelson \\ Françoise Degoul \\ Cécile Marsac \\ Gérard Ponsot \\ Patrick Lestienne}

\section{ADRESSES}

I. Nelson: stagiaire de recherche. P. Lestienne; directeur de recherche à l'Inserm. Inserm U. 298, CHR d'Angers, 49033 Angers Cedex, France.

F. Degoul : stagiaire de recherche. C. Marsac: docteur en médecine. Inserm U.75, CHU Necker, 75730 Paris Cedex, France.

G. Ponsot : docteur en médecine, professeur agrégé en neuropédiatrie. Hôpital Saint-Vin- cent de Paul, 75674 Paris Cedex 14, France. e syndrome de KearnsSayre est une affection rare, d'évolution lentement progressive, débutant à des âges variés mais souvent dans l'enfance, pluritissulaire, touchant principalement le système musculaire mais aussi le système nerveux central et les organes neuro-sensoriels.

Ce syndrome a été décrit pour la première fois par T.P. Kearns et G.P. Sayre en 1958 [1] chez deux enfants présentant une ophtalmoplégie externe progressive, une rétinite pigmentaire et des troubles de la conduction myocardique à type de bloc auriculo-ventriculaire. Par la suite, de nombreuses autres observations de syndrome de Kearns-Sayre furent rapportées aussi bien chez l'adulte que chez l'enfant, et il s'avéra qu'à cette triade symptomatique s'associait de façon très fréquente un retard statural, une ataxie ${ }^{*}$, un retard intellectuel, d'autres troubles neurosensoriels à type de surdité, une hyperprotéinorachie transsudative ${ }^{* *}$ et des anomalies endocriniennes diverses. A côté des syndromes de Kearns-Sayre typiques, il existe des formes incomplètes [2] comprenant toujours une ophtalmoplégie externe progressive, mais où il man-

\footnotetext{
* Ataxie : trouble de la coordination des mouvements volontaires.

* Hyperprotéinorachie transsudative: aug mentation de la concentration en protéine du liquide céphalo-rachidien, sans syndrome inflammatoire.
} 
que sojt. la rétinopathie, soit les troubles de conduction cardiaque, soit les deux; ces formes incomplètes ont surtout été décrites chez l'adulte. Le point commun à tous ces tableaux cliniques est la présence lors de l'étude histologique des biopsies musculaires de fibres anormales (ragged-red-fibers), fibres musculaires rouges en lambeaux qui témoignent d'une anomalie mitochondriale et qui sont utiles pour le diagnostic malgré leur absence de spécificité.

Il est prouvé maintenant que le syndrome de Kearns-Sayre fait partie des maladies mitochondriales dues à un dysfonctionnement de la chaîne respiratoire mitochondriale.

\section{Les grands principes du métabolisme énergétique mitochondrial}

La mitochondrie est la voie finale commune des réactions cataboliques des glucides, des protides et des lipides, où l'énergie est transformée au niveau de la chaîne respiratoire qui comporte cinq complexes localisés dans la membrane mitochondriale interne (figure 1). L'énergie libérée par l'oxydation des composés réduits (NADH, FADH) est utilisée pour synthétiser l'ATP par l'intermédiaire d'un gradient de protons dû au transfert d'électrons à travers la chaîne respiratoire qui pompe les protons de la matrice vers la face cytoplasmique de la membrane mitochondriale interne. Les protons sont « pompés » au niveau de trois sites : complexes I, III, IV. L'énergie contenue dans le gradient de protons est utilisée pour synthétiser, grâce à un phénomène de couplage, de l'ATP à partir de l'ADP au niveau du «canal protonique» de l'ATP synthétase (complexe V) lorsque les protons refluent de l'espace intermembranaire vers la matrice.
Les différents complexes de la chaîne respiratoire peuvent être maintenant étudiés assez précisément par les méthodes enzymologiques et oxygraphiques [3]. Quand une anomalie a été identifiée, on peut étudier les sous-unités de ce complexe après électrophorèse sur gel bidimentionnel de polyacrylamide des préparations mitochondriales et immunotransfert avec des anticorps purifiés spécifiques d'holoenzymes et des sous-unités héminiques [3].

La particularité unique de la mitochondrie est qu'elle a son propre ADN qui a la forme d'un double brin circulaire comprenant 16569 paires de base qui ont été complètement séquencées par l'équipe de Sanger en 1981 [4] (figure 2). Cet ADN mitochondrial a besoin de facteurs nucléaires pour sa réplication, sa transcription et sa traduction. Il existe plusieurs copies d'ADN par mitochondrie (deux à dix copies) [5].

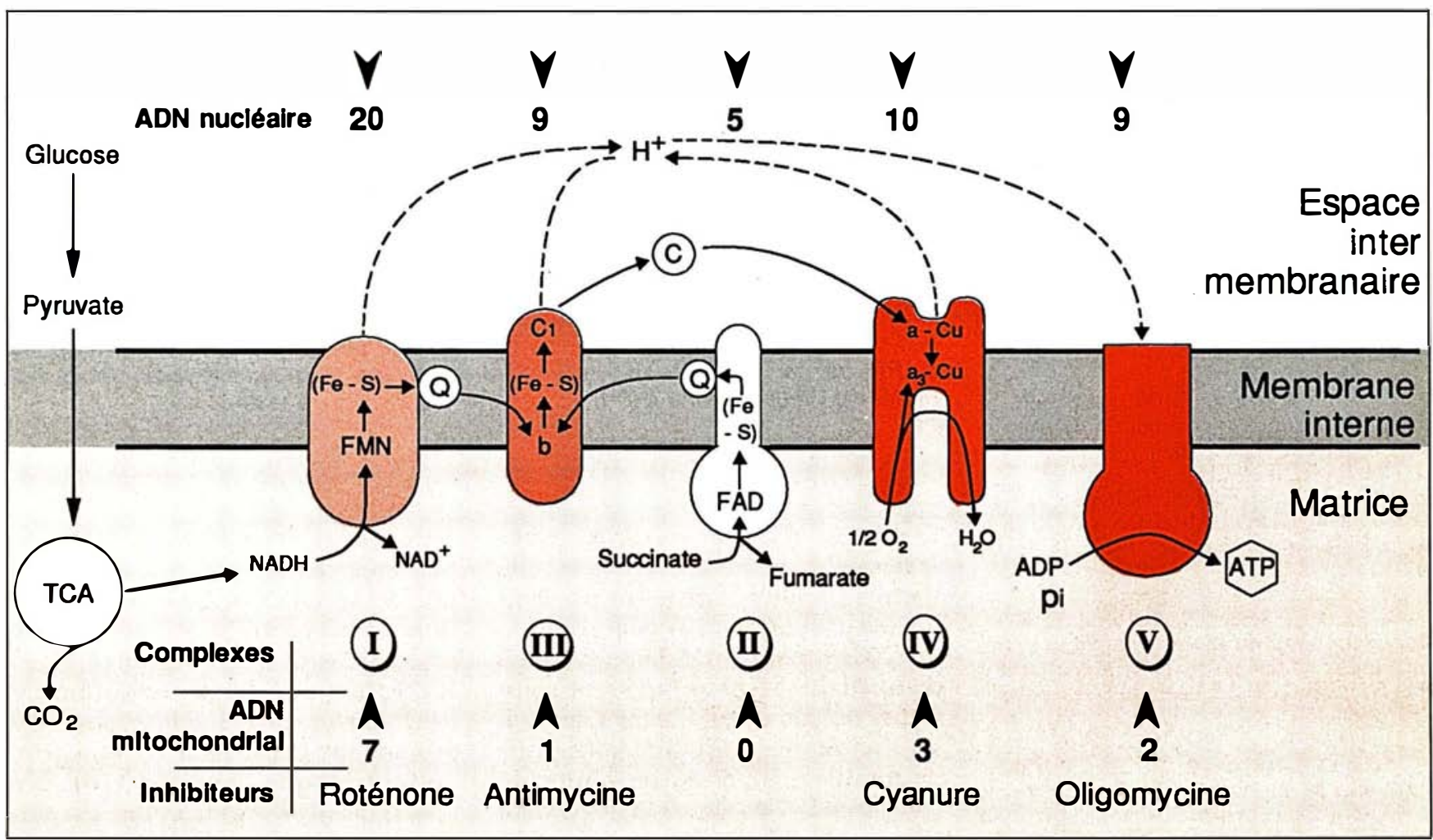

Figure 1. Représentation simplifiée des cinq complexes de la chaîne respiratoire indiquant l'origine génétique des protéines les composant, le principe général de la phosphorylation oxydative et les inhibiteurs des divers

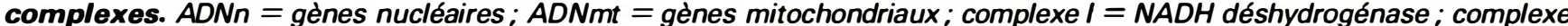
$\|$ = succínate déshydrogénase ; complexe $I I I=$ ubiquinone cytochrome $c$ réductase ; complexes $N=$ cytochrome $C$ oxydase ; complexe $V=A T P$ synthétase $; Q=$ ubiquinone $C=$ cytochrome $C ; T C A=$ cycle tricarboxylique (Krebs). 


\section{RÉFÉRENCES}

1. Kearns TP, Sayre O. Retinitis pigmentosa, external ophthalmoplegia and complete heart block. AMA Arch Ophthalmol 1958; 60 : 280-9.

2. Petty RKH, Harding AE, Morgan-Hughes JA. The clinical features of mitochondrial myopathy. Brain 1986; 109 : 915-38.

3. Darley-Usmar VM, Rickwood D, Wilson MT. Mitochondria. A practical approach. London : Irl Press, 1987 : 321

4. Anderson S, Bankier AT, Barrel BG, et al. The nucleotide sequence of the human mitochondrial DNA. Nature 1981 ; 290 : 457-65.

5. Bogenhagen D, Clayton DA. The number of mitochondrial deoxyribonucleic acid genomes in mouse $\mathrm{L}$ and human HeLa cells. J Biol Chem 1974; 249 : 7991-5.

6. Bresolin N, Moggio M, B et L, et al. Progressive cytochrome c oxidase deficiency in a case of Kearns-Sayre syndrome : morphological, immunological and biochemical studies in muscle biopsies and autopsy. Ann Neurol $1987 ; 21: 564-72$.

7. Holt IJ, Harding AE, Morgan-Hugues $\mathrm{H}$. Deletion of muscle mitochondrial DNA in patients with mitochondrial myopathies. Nature 1988 ; 331 : 717-9.

8. Lestienne P, Ponsot G. Kearns-Sayre syndrome with muscle mitochondrial DNA deletion. Lancet 1988; $1: 885$.

9. Zeviani M, Servidei S, Gellera C, et al. Deletions of mitochondrial DNA in KearnsSayre syndrome. Neurology $1988 ; 38$ : 1339 . 46.

10. Saiki RK, Scharf S, Faloona F, et al. Enzymatic amplification of $\beta$-globin genomic sequences and restriction site analysis for diagnosis of sickle cell anemia. Science 1985 ; 230 : $1350-4$.

11. Schon EA, Rizzuto R, MoraesCT, Nakase $\mathrm{H}$, Zviani M, DiMauro S. A direct repeat is a hot spot for large-scale deletion of human mitochondrial DNA. Science 1989 ; 244 : 346 -

12. Poulton J, Deadman ME, Gardiner RM Duplications of mitochondrial DNA in mito-

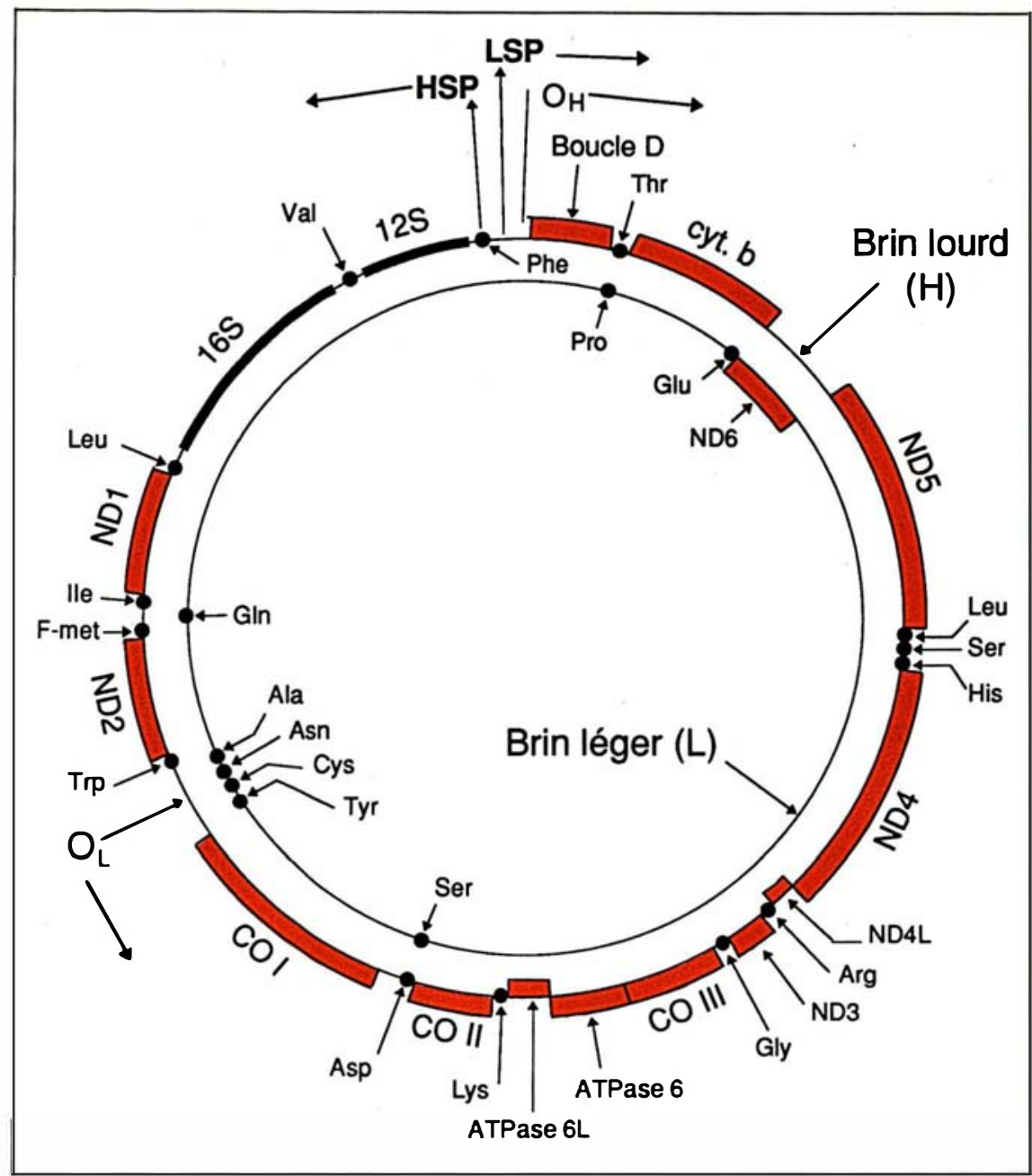

Figure 2. Organisation du génome mitochondrial humain. La séquence fut établie par F. Sanger et son équipe (Anderson et al., 1981). HSP: promoteur du brin lourd (heavy strand promoter); $L S P=$ promoteur du brin léger (light strand promoter); boucle $D=$ boucle de déplacement ; $\mathrm{OH}=$ origine de réplication du brin lourd; $O L=$ origine de réplication du brin léger; $12 \mathrm{~S}$ et $16 \mathrm{~S}=$ gènes des ARN ribosomiques mitochondriaux : $C O=$ cytochrome $C$ oxydase $: N D=N A D H$ désydrogénase; $A T P a s e=A T P$ synthétase. Les points représentent des gènes d'ARNt qui ponctuent les gènes mitochondriaux (en rouge, gènes codant pour des protéines. Seul un gène (ND6) est représenté sur le brin léger.

Il n'a pas d'intron et est d'origine maternelle, car les mitochondries du spermatozoïde ne pénètrent pas dans l'ovule. Il comprend 37 gènes dont 13 qui codent pour 13 des 66 sous-unités de la chaine respiratoire : sept sousunités du complexe I, trois sous-unités du complexe IV, une sous-unité du complexe III, deux sous-unités du complexe V. Les autres gènes codent pour deux ARN ribsomiques et 22 ARN de transfert. Les autres sousunités sont codées par l'ADN nucléaire (figure 1).

Il existe une région non codante de
700 paires de bases environ délimitée par les gènes $\mathrm{ARNt}{ }^{\text {Pro }}$ et $\mathrm{ARNt}$ Phe qui comprend l'origine de réplication du brin lourd $(\mathrm{OH})$ et les deux promoteurs de transcritpion (HSP et LSP) (figure 2). La transcription de l'ADNmt se fait à partir de deux promoteurs produisant des transcrits polycistroniques ponctués par des pré-ARNt qui seront clivés par des ribonucléases ; les ARN monocistroniques ainsi libérés sont ensuite polyadénylés et les ARNt subissent une maturation par l'addition d'une séquence CCA à l'extrémité 3 ' cataly- 
sée par l'ARNt nucléotidyl-transférase. La traduction des ARN mitochondriaux fait intervenir un code génétique particulier (Tableau I).

\section{Phosphorylation oxydative et syndrome de Kearns-Sayre}

Une anomalie de fonctionnement de la chaîne respiratoire est actuellement établie dans ce syndrome. Elle peut être suspectée par des examens biologiques simples : hyperlactacidémie et surtout hyperlactacidorachie, augmentation des rapports Redox ${ }^{*}$ : $\beta$-hydroxybutyrate/acéto-acétate, lactate/pyruvate, et par l'augmentation des acétyl-carnitines urinaires.

Les méthodes enzymologiques et oxygraphiques ont mis en évidence des déficits partiels et multiples des complexes de la chaîne respiratoire touchant principalement les complexes I et IV [2, 6].

L'anomalie du métabolisme mitochondrial dans ce syndrome peut être également mise en évidence par des méthodes histochimiques : l'observation des biopsies musculaires fait apparaître par la coloration du trichrome Gomori des ragged-red-

* Rapports d'oxydoréduction, appréciés par le rapport entre les formes réduites et les formes oxydées des substrats et produits d'une même réaction réversible d'oxydoréduction (par exemple, lactate-pyruvate). fibers, et, plus récemment, les colorations histochimiques de la cytochrome c-oxydase ont montré au niveau du tissu musculaire un aspect en mosaïque, comprenant des fibres blanches et des fibres hypercolorées, tout à fait évocateur de cette maladie. L'étude en microscopie électronique révèle des anomalies structurales des mitochondries: désorganisation des crêtes, crêtes enroulées, et parfois inclusions paracristallines.

\section{Des délétions de I'ADN mitochondrial}

L'origine de ce syndrome reste encore mystérieuse. Toutefois, la découverte récente de délétions de l'ADN mitochondrial dans cette affection ouvre des perspectives intéressantes pour la compréhension de cette maladie. Ce sont Holt et al. [7] qui, les premiers, ont trouvé des délétions de l'ADN mitochondrial dans des myopathies qui comportaient toutes une ophtalmoplégie progressive externe et, à peu près à la même époque, Lestienne et al. [8] ont décrit la première délétion dans un syndrome de Kearns-Sayre complet de l'enfant (figure 3). L'existence de ces délétions dans le syndrome de Kearns-Sayre a ensuite été confirmée par d'autres équipes, en particulier par Zeviani et al. [9] qui l'ont retrouvée dans sept cas sur sept de syndrome de KearnsSayre typique.

\begin{tabular}{|c|c|c|c|}
\hline \multicolumn{4}{|c|}{$\begin{array}{c}\text { Tableau I } \\
\text { CODE GÉNÉTIQUE SPÉCIFIQUE }\end{array}$} \\
\hline AUX DIFFÉRENTES ESPËCES DE MITOCHONDRIES \\
\hline UGA & Code universel & $\begin{array}{c}\text { Code } \\
\text { mit. levure }\end{array}$ & $\begin{array}{c}\text { Code } \\
\text { mit. mammifères }\end{array}$ \\
\hline AUA & Stop & Trp & Trp \\
\hline CUA & Leu & Met & Met \\
\hline AGA & Arg & Thr & Leu \\
\hline AGG & Arg & Stop \\
\hline
\end{tabular}

Ile $=$ isoleucine $;$ Leu $=$ leucine $;$ Arg $=$ arginine $; \operatorname{Trp}=$ tryptophane $;$ Met $=$ méthionine Thr = thréonine; Stop = codon d'arrêt de la traduction.
La plupart des équipes ont mis en évidence ces délétions de l'ADN mitochondrial par la méthode de Southern, le plus souvent à partir de l'ADN total. L'ADN mitochondrial est linéarisé par une enzyme de restriction qui coupe la molécule en un seul endroit comme le Pvu-II (enzyme qui clive à la position 2650), puis est soumis à une électrophorèse sur gel d'agarose à $0,8 \%$, transféré sur un filtre de nylon et hybridé avec de l'ADNmt marqué au ${ }^{32} \mathrm{P}$. Cette technique a permis de détecter deux populations d'ADNmt, l'une normale de 16,7 kpb (kilopaires de bases) et l'autre plus courte, de longueur variable correspondant à la molécule d'ADNmt délété. L'utilisation combinée de sondes variées d'ADNmt et d'enzymes de restriction judicieusement choisies a permis de mesurer et de localiser la délétion. La proportion des molécules d'ADNmt délété par rapport aux molécules normales est mesurée par densitométrie des fragments hybridés. Cette technique ne permet pas de mettre en évidence des petites délétions au niveau de l'ADNmt. La méthode d'amplification in vitro PCR (polymerase chain reaction) [10] permettra, associée au séquençage de localiser avec plus de précision les petites délétions et de détecter des délétions de quelques paires de bases.

Caractéristiques des délétions. Elles sont retrouvées dans la presque totalité des cas de syndrome de KearnsSayre sauf quatre [7, 11] et dans la moitié des cas de myopathie avec ophtalmoplégie externe progressive [7].

Une anomalie mitochondriale particulière à type de duplication a été trouvée chez deux patients atteints de myopathie avec ophtalmoplégie externe progressive [12] .

Elles ont une longueur variable d'un cas à l'autre, comprise entre 2,5 et $8 \mathrm{kpb}$, mais ont la même longueur chez le même malade. Elles respectent dans la totalité des cas la région comportant l'origine de réplication du brin lourd, les deux promoteurs de transcription des chaînes légères et lourdes et les gènes d'ARN ribosomiques $12 S$ et $16 S$. Dans la presque totalité des cas $[9,11]$, les délétions respectent également d'origine de réplication du brin léger (figure 4). 


\section{RÉFÉRENCES}

13. Wrischnik LA, Higuchi RG, Stoneking $\mathrm{M}$, et al. Length mutations in human mitochondrial DNA : direct sequencing of enzymatically amplified DNA. Nucleic Acids Res 1987 ; 15 : $529-41$.

14. Boursot $\mathrm{P}$, Yanekawa $\mathrm{H}$, Bonhomme $\mathrm{F}$ Deletion in mouse mitochondrial DNA. Mol Biol Evol 1987 ; 4 : 46-55.

15. Clayton DA. Transcription of the mammalian mitochondrial genome. Annu Rev Biochem 1984 : 53 : 573-94.

16. Byrne E, Marzuki S, Sattayasai N, Dennett X, Trounce I. Mitochondrial studies in Kearns-Sayre syndrome: normal respiratory chain function with absence of a mitochondrial translation product. Neurology 1987; $37: 1530-4$.

17. Wallace DC. Maternal genes : mitochondrial diseases. Birth defects, vol. 23, n० 3 . New York: Alan R Liss, 1987 : 137-90.

18. Rowland LP, Hausmanowa-Petrusewicz I, Bardurska B. Kearns-Sayre syndrome in twins: lethal dominant mutation acquired disease? Neurology $1988 ; 38: 1399-402$.

19. Ozawa T, Yoneda M, Tanaka M, et al. Maternal inheritance of deleted mitochondrial DNA in a family with mitochondrial myopathy. Biochem Biophys Res Commun 1988 ; $154: 1240-1247$.

20. Zeviani M, Italy M, Servidei S, et al. Dominantly inherited progressive ophthalmoplegica with large deletions of mitochondrial DNA. Neurology 1989; 39 : 256-7.

21. Rötig A, Bonnefont JP, Colonna M, et al. Les remaniements du génome mitochondrial dans les déficits énergétiques de l'enfant.

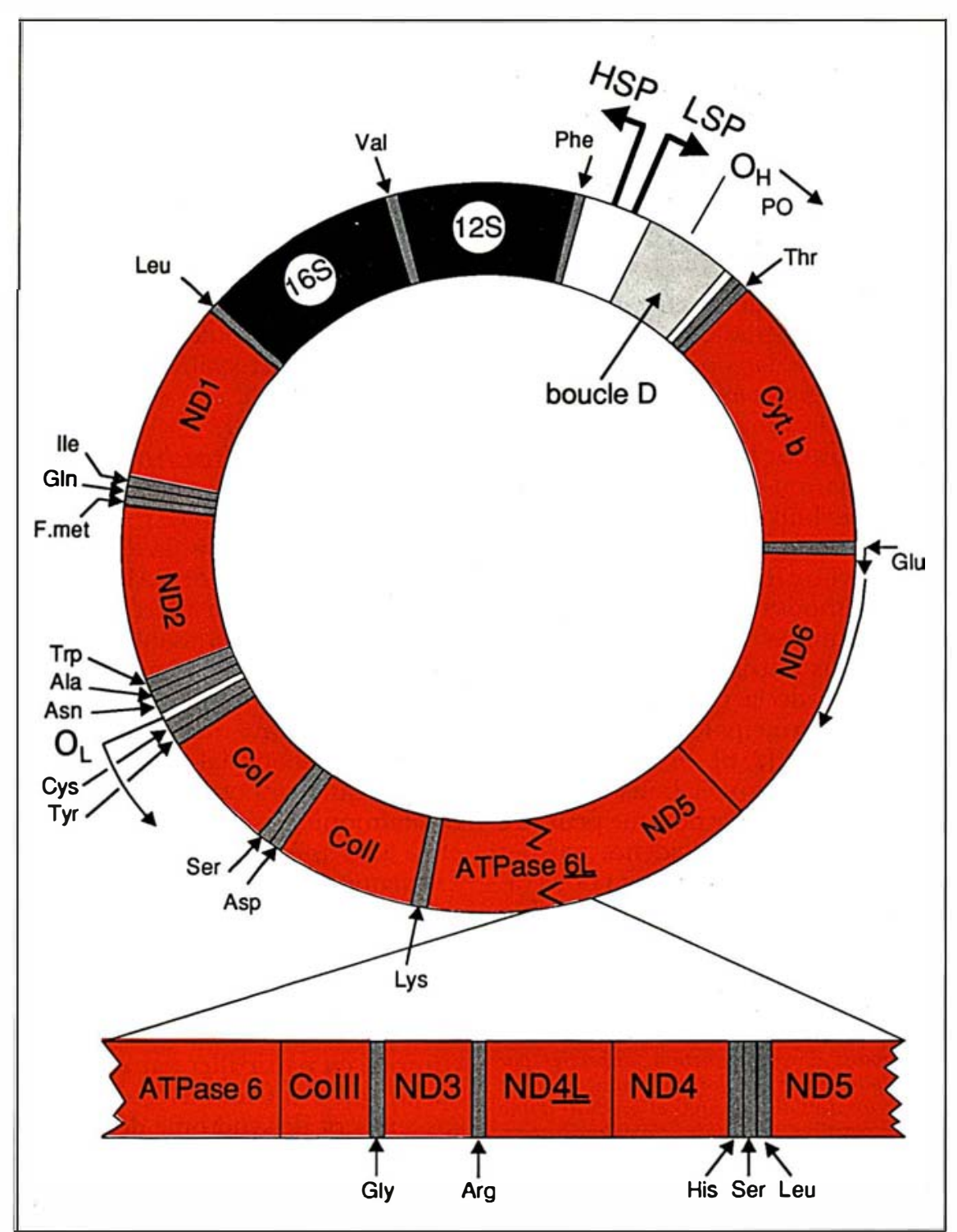

Figure 3. Délétion de I'ADN mitochondrial observée chez le patient présentant le syndrome de Kearns-Sayre décrit dans [8]. En rouge, les gènes codant pour les protéines; en gris, les gènes des ARNt ponctuateurs; en noir, les gènes ribosomiques. Les flèches se trouvant devant les gènes d'ARNt indiquent s'ils sont transcrits à partir du brin L (sens des aiguilles d'une montre) ou du brin $H$ (sens inverse des aiguilles d'une montre). Seul le gène ND6 est transcrit à partir du brin $L$, ce qui est indiqué par une flèche.

Dans près de 30 à $40 \%$ des cas de syndrome de Kearns-Sayre et de myopathie avec ophtalmoplégie externe progressive, elles sont situées dans une zone préférentielle dite de «point chaud» (hot spot), entre $8200 \pm 400$ et $13400 \pm 400$ (paires de base). Cette région, qui au cours de la réplication de l'ADNmt est exposée en simple brin, pourrait contenir des sites fragiles facilitant les délétions.

Au début, on a pensé que les délé- tions de l'ADNmt étaient préférentiellement localisées dans le tissu musculaire, mais elles ont été également retrouvées dans les lymphocytes, les fibroblastes après un temps de culture court, le système nerveux central et la moelle. Elles sont hétéroplasmiques, c'est-à-dire qu'outre les molécules d'ADNmt délétées, il existe des molécules de longueur normale. Le rapport entre la molécule normale et la molécule délétée varie de 0,3 à 4 selon les malades [9] 
et, chez un même malade, ce rapport peut varier suivant les différents tissus [9] .

Conséquences des délétions. Toutes les équipes qui travaillent sur l'ADNmt $[8,9,11]$ dans le syndrome de Kearns-Sayre ont noté qu'il n'y avait pas de corrélation étroite entre la taille, la localisation de la délétion et les phénotypes cliniques et biologiques.

Actuellement, on est réduit à un certain nombre d'hypothèses pour expliquer ces phénomènes.

- Délétion de l'ADNmt et pathologie. Il paraît certain que ces délétions sont pathologiques, en effet les seules délétions qui ont déjà été trouvées chez des sujets asymptomatiques sont très rares, de très petite taille (de l'ordre de $10 \mathrm{pb}$ ) et sont localisées à des régions non codantes [13]. Toutefois, il a été rapporté des larges délétions de $5 \mathrm{kpb}$ touchant des régions codantes ( $A R N$ t et gènes codant pour les sous-unités des complexes de la chaîne respiratoire) hétéroplasmiques avec un pourcentage élevé de molécules délétées ( $80 \%$ ) chez des souris ne présentant aucune pathologie apparente particulière [14].

- Transcription polycistronique de l'ADNmt. La transcription étant polycistronique* $^{*}$ [15], on peut imaginer qu'une délétion de l'ADNmt, quelle que soit sa localisation, puisse avoir de sérieuses conséquences sur la traduction de gènes non compris dans cette délétion, ce qui peut expliquer, par exemple, que certains malades aient une réduction d'au moins $50 \%$ de l'activité de leur complexe IV (cytochrome c-oxydase), alors que la délétion épargne les gènes codant pour les trois sousunités de ce complexe [9].

- Fusion des bords de la délétion de l'ADNmt. Protéine hybride. Comme le montre la figure 5, la jonction des bords de la délétion du patient décrit dans l'article cité [8], recrée un codon stop pour l'ATPase 8 (TAG $\rightarrow$ TAA) et permet de produire une nouvelle protéine de fusion normalement

\footnotetext{
* Polycistronique : transcription de plusieurs gènes(cistrons) en un seul $A R N$, qui sera soit clivé en plusieurs messagers (cas de la mitochondrie), soit sans clivage, traduit en plusieurs protéines (cas des bactéries).

$\mathrm{m} / \mathrm{s} n^{\circ} 7$ vol. 5 , septembre 89
}

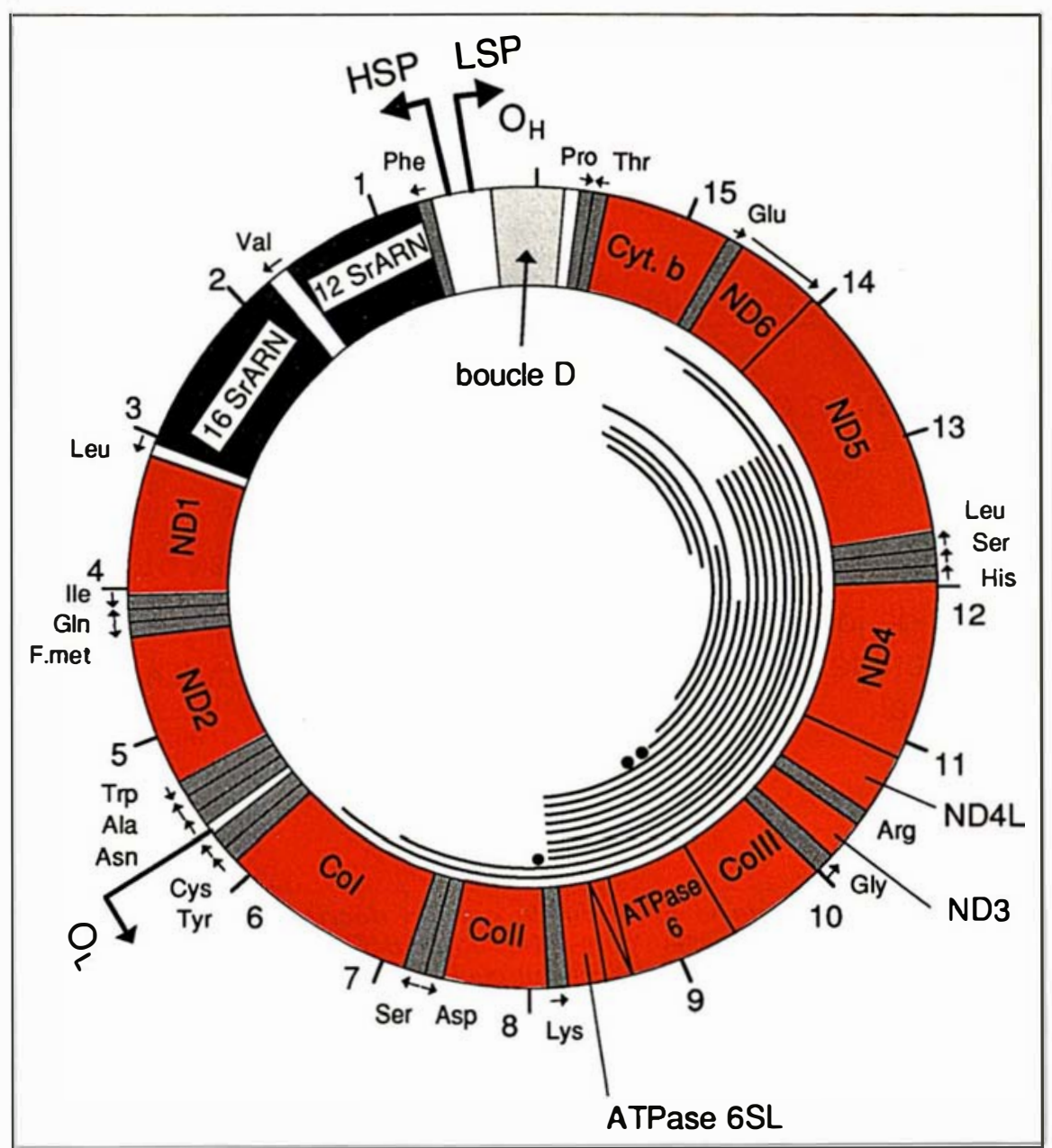

Figure 4. Résumé des 15 délétions trouvées dans le muscle ou dans les

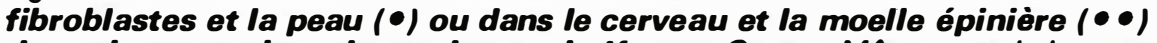
de patients atteints du syndrome de Kearns-Sayre. Mêmes symboles que sur la figure 3.

absente ATPase-6-ND5. En effet, la fusion observée est en phase de lecture, ce qui permettrait sa transcription et sa traduction en une protéine hybride qui possède l'extrémité $\mathbf{N}$ terminale de la protéine ATPase 6 et la moitié C-terminale de la protéine ND5. Cette nouvelle protéine pourrait entrer en compétition avec les autres sous-unités de la chaîne respiratoire dans l'édification des différents complexes et entraîner ainsi un dysfonctionnement plus ou moins sévère de l'oxydoréduction mitochondriale. On peut imaginer qu'en fonction de la localisation, de la taille de la délétion, il y aura formation de protéines de fusion différentes [16] qui pourront perturber de façon variable le fonctionnement de la chaîne respiratoire. Ces protéines de fusion ou hybrides n'ont pas été encore mises en évidence dans le muscle ou dans d'autres organes. - Complémentation entre les molécules d'ADNmt délété et les molécules d'ADNmt normal. A l'état normal [17], il y a une complémentation entre les molécules d'ADNmt et également entre les molécules d'ADNmt et d'ADN nucléaire. En effet, l'ADNmt a besoin pour sa réplication et sa transcription d'enzymes codées par le génome nucléaire.

La persistance à une forte proportion de molécules d'ADNmt délétées nécessite obligatoirement une complémentation par l'ADNmt normal. 


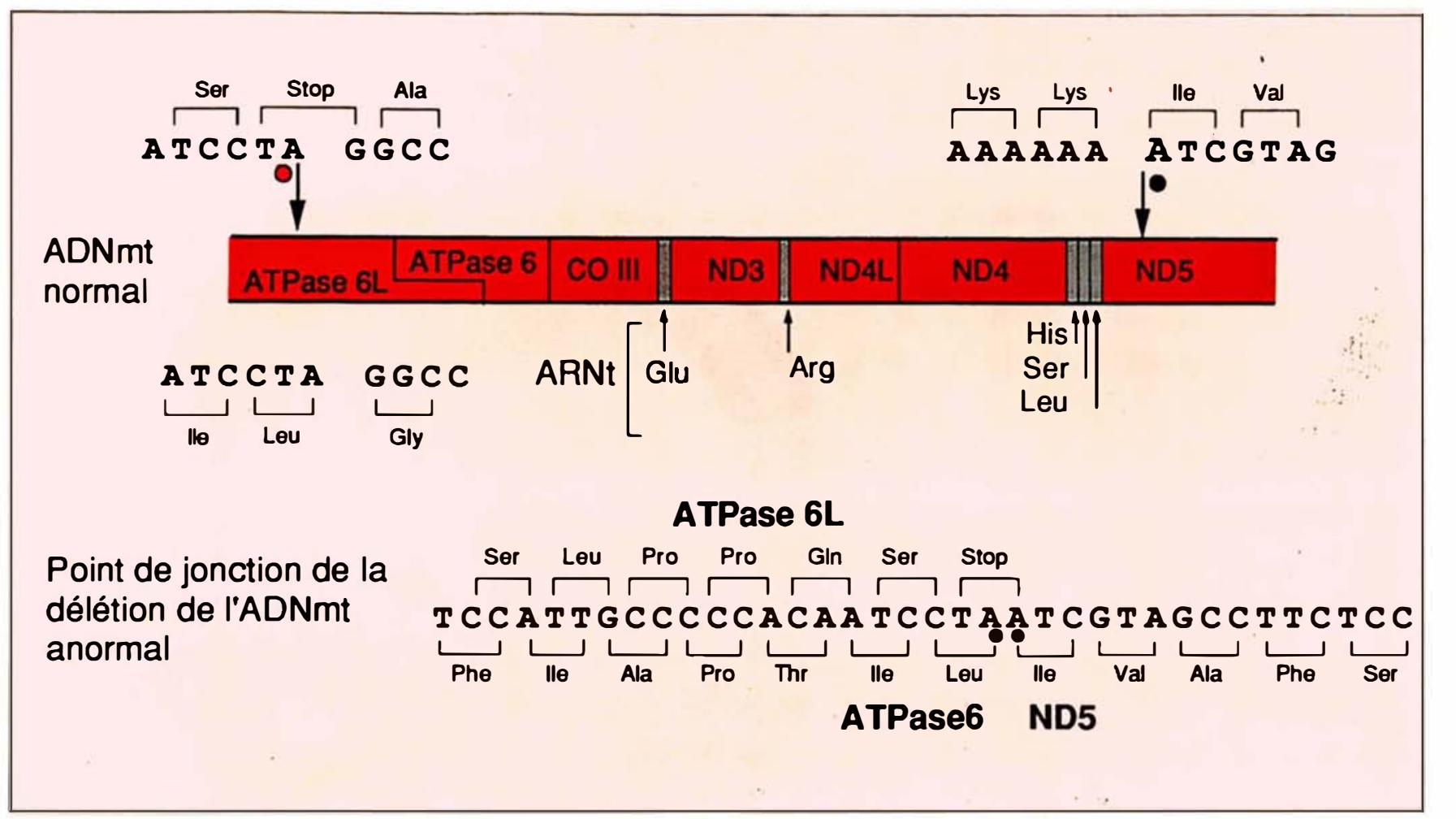

Figure 5. Jonctions de la délétion du patient décrit dans l'article cité en [8]. La taille exacte de la délétion est de 4,666 kpb en accord avec la figure 3. Nucléotide 8571 : point rouge; nucléotide 13237: point noir. Les gènes de l'ATPase AGL [8] et de l'ATPase 6 sont légèrement chevauchants et sont lus dans deux phases de lecture différentes se terminant par Ser-Stop (TAG) pour I'ATPase 6L. Après délétion, le codon TAG de terminaison qui suit la sérine carboxyterminale de I'ATPase 6L devient TAA... qui est un autre codon de terminaison. La phase de lecture des parties non délétées des gènes ATPase 6 et ND5 n'est pas modifiée, aboutissant à la possible synthèse d'une protéine hybride.

Il est vraisemblable que l'expression du phénotype des maladies mitochondriales comportant une délétion de l'ADNmt n'apparaît qu'à partir d'une certaine quantité (seuil minimal) de molécules d'ADNmt délété [17] .

Les effets de la complémentation sur l'expression du phénotype pathologique peuvent varier en fonction de plusieurs facteurs: (1) un facteur quantitatif, le rapport entre le nombre de molécules d'ADNmt délétées et le nombre de molécules d'ADNmt normales ; (2) la taille et la localisation de la délétion au niveau de l'ADNmt délété ; (3) la localisation des molécules d'ADNmt délétées et d'ADNmt normales ; les effets peuvent être différents selon que la complémentation est intermitochondriale, les deux types de molécules d'ADNmt étant dans des mitochondries différentes, ou intramitochon- driale, les deux types de molécules d'ADNmt étant dans la même mitochondrie. Ce dernier type de complémentation (complémentation intramitochondriale) paraît le plus vraisemblable. En effet, les molécules délétées peuvent se répliquer et être transcrites puisque la délétion respecte cette région; en revanche, les transcrits ne pourraient être traduits en l'absence d'ARNt provenant des molécules d'ADN normal, beaucoup d'ARNt indispensables à la traduction étant inclus dans la délétion.

Si cette complémentation a des effets bénéfiques, on ne peut écarter qu'elle ait également des conséquences nocives, car elle permettrait la traduction de protéines anormales provenant de la molécule d'ADNmt délétée et il n'est pas exclu que l'expression de cet ADN délété perturbe celle de l'ADNmt normal.

- Ségrégation mitotique précoce. Le phénotype pathologique peut également varier en fonction d'une ségrégation préférentielle des mitochondries à ADNmt délété suivant les différents tissus [17]. Ainsi le phénotype myopathie avec ophtalmoplégie progressive isolée peut être dû à une ségrégation mitotique précoce dans l'ovocyte ou le zygote, conduisant à une distribution préférentielle des mitochrondries à $\mathrm{ADN}$ mt délété dans le tissu musculaire. Ceci demande à être confirmé par la recherche de délétion de l'ADNmt dans les différents tissus des malades présentant ce phénotype.

Origine des délétions de l'ADNmt. Le syndrome de Kearns-Sayre a tout d'abord été considéré comme secondaire à une mutation autosomique dominante létale, c'est-à-dire ne permettant pas aux sujets atteints de se reproduire [18]. La découverte de délétions de l'ADNmt dans cette 
maladie et dans une grande partie des myopathies mitochondriales avec ophtalmoplégie externe progressive conduit à proposer d'autres hypothèses quant aux origines de ces maladies : (1) ces délétions peuvent provenir d'une délétion préexistante chez la mère, puisque l'ADNmt est d'origine maternelle; (2) ou être acquise très précocément durant les premiers stades de l'embryogenèse puisque ces délétions sont pluritissulaires.

La première possibilité paraît moins vraisemblable. La majorité des syndromes de Kearns-Sayre sont en effet sporadiques et, en particulier, les mères des enfants atteints ne présentent aucun signe pathologique [2]; ceci n'exclut pas toutefois qu'elles puissent présenter des délétions d'ADNmt, mais en proportion inférieure au seuil minimal requis pour permettre l'expression du phénotype pathologique. Les études en Southern de l'ADNmt musculaire des mères n'ont pas montré de délétion [8] , en dehors de l'observation rapportée par Ozawa et al. [19] qui a montré chez la mère et sa fille atteinte d'ophtalmoplégie externe progressive, des délétions de l'ADNmt musculaire de taille différente mais ayant une partie commune de 1,2 kpb.

Très récemment, Zeviani et al. [20] ont rapporté, sur plusieurs générations dans une même famille, des individus ayant une ophtalmoplégie progressive externe avec myopathie proximale, dysphagie, de début tardif et se transmettant sur un mode dominant autosomique. Et ils ont trouvé chez deux malades de cette famille qu'ils ont étudiée (un homme et sa tante maternelle) une délétion de 8 et de $8,5 \mathrm{kpb}$ semblable mais non identique. Ces auteurs ont suggéré que cette maladie serait due à une mutation dominante d'un gène nucléaire codant pour une molécule impliquée dans la réplication de l'ADNmt.

Le caractère acquis de la grande majorité de ces maladies correspond cependant mieux à la réalité. Toutefois, actuellement, aucune étiologie précise n'a pu être mise en évidence. Rowland [18] avait proposé une origine virale au syndrome de KearnsSayre (rétrovirus, infection virale persistante, etc.). Très récemment, l'amplification de la séquence jointive de la délétion a pu être réalisée chez un $m / s n^{\circ} 7$ vol. 5 , septembre 89 malade décrit dans [8] et n'a pas mis en évidence d'insertion étrangère (ADN viral, par exemple).

La découverte par Schon et al. [11], après amplification, de séquences répétitives de $13 \mathrm{pb}$ de part et d'autre des délétions de $5 \mathrm{kpb}$ trouvées dans la zone préférentielle décrite ci-dessus conduit à penser qu'elles pourraient se produire lors de phénomènes de recombinaison de l'ADNmt.

\section{Perspectives diagnostiques et thérapeutiques}

Les maladies mitochondriales avec délétion de l'ADNmt ont une expression phénotypique pluritissulaire essentiellement neuromusculaire dont la forme clinique la plus complète est réalisée par la syndrome de Kearns-Sayre et dont le dénominateur commun est l'ophtalmoplégie progressive externe mais d'autres cas cliniques ont été décrits (maladie de Pearson [21]. Le ou les mécanismes par lequel les délétions de l'ADNmt entraînent ces manifestations cliniques ainsi que leur origine ne sont pas actuellement connus et, plus encore, on ne sait pas si ces délétions sont la cause ou la conséquence de ces maladies.

La mise en évidence des délétions par la technique de Southern est relativement simple et ne nécessite que peu de matériel $(50 \mathrm{mg}$ de tissu musculaire, culture de fibroblastes, lymphocytes). La méthode d'amplification in vitro (PCR) [10] va permettre d'identifier avec beaucoup plus de précision la délétion.

Il n'existe pas encore de thérapeutique spécifique de ces maladies mitochondriales avec délétion de l'ADNmt. Certaines améliorations ont été rapportées dans le syndrome de Kearns-Sayre avec le co-enzyme $Q$ et la riboflavine en cas de déficit en complexe I et avec la vitamine $\mathrm{C}$ et la ménadione dans un syndrome de Kearns-Sayre avec déficit en complexe III.

On peut espérer que l'apport de composés substitutifs de l'ATP, au moyen de liposomes ou en jouant sur l'expression préférentielle de l'ADNmt normal, pourrait remédier au déficit énergétique cellulaire responsable de ces affections sévères

\section{Summary}

Mitochondrial DNA deletions in Kearns-Sayre syndrome and in myopathies with progressive external ophthalmoplegia

Kearns-Sayre syndrome associates a myopathy with progressive external ophthalmoplegia, a pigmentary retinopathy and a cardiac block branch. Simpler forms mainly involve external ophtalmoplegia. Histochemistry reveals the constant presence of abnormal (ragged red) muscle fibers. These diseases generally appear spontaneously and are associated with mitochondrial DNA deletions which are different between individuals but similar within individuals. These mitochondrial DNA deletions induce biochemical abnormalities of the mitochondrial metabolism by energy production defect via oxidative phosphorylation reactions.

\section{TIRÉS A PART}

\section{Nelson.}

\begin{tabular}{|l|}
\hline La mutation responsable \\
du phénotype mdx de la \\
souris (modèle de myopa- \\
thie bénigne avec absence \\
dedystrophine) est l'appa- \\
rition d'un codon stop \\
remplaçant un codon glu- \\
tamique (CAA - TAA) en un \\
point correspondant aux \\
$27 \%$ aminoterminaux de \\
la protéine (Sincinski et \\
al., Cambridge, GB-Science \\
$1989 ; 244: 1578-80$ ). \\
\hline
\end{tabular}

